\title{
PSEUDO-REPRESENTATIONS OF WEIGHT ONE ARE UNRAMIFIED
}

\author{
FRANK CALEGARI AND JOEL SPECTER
}

\begin{abstract}
We prove that the determinant (pseudo-representation) associated to the Hecke algebra of Katz modular forms of weight one and level prime to $p$ is unramified at $p$.
\end{abstract}

\section{CONTENTS}

1. Introduction

2. Determinants

2.1. Determinants of degree $d=2$

2.2. Ordinary Determinants

3. Galois Deformations

References

\section{INTRODUCTION}

Let $p$ be prime, and let $N \geq 5$ be prime to $p$. Let $\mathcal{O}$ be the ring of integers in a finite extension $K$ of $\mathbf{Q}_{p}$ with uniformizer $\varpi$. Let $X_{1}(N)$ be the modular curve considered as a smooth proper curve over $\operatorname{Spec}(\mathcal{O})$, and let $\omega$ be the pushforward of the relative dualizing sheaf along the universal elliptic curve. The coherent cohomology group $H^{0}\left(X_{1}(N)\right.$, $\left.\omega\right)$ may be identified with the space of modular forms of weight one with coefficients in $\mathcal{O}$. For general $m$, one knows that the map:

$$
H^{0}\left(X_{1}(N), \omega\right) \rightarrow H^{0}\left(X_{1}(N), \omega / \varpi^{m}\right)
$$

need not be surjective. This was first observed by Mestre for $N=1429$ and $p=2$, (see Edi06, Appendix A]), and many examples for larger $p$ have been subsequently computed by Buzzard and Schaeffer [Buz14, Sch15]. In particular, if $\mathbf{T}$ denotes the subring of

$$
\operatorname{End}_{\mathcal{O}} \lim _{\rightarrow} H^{0}\left(X_{1}(N), \omega / \varpi^{m}\right)=\operatorname{End}_{\mathcal{O}} H^{0}\left(X_{1}(N), \omega \otimes K / \mathcal{O}\right),
$$

generated by Hecke operators $T_{l}$ and $\langle l\rangle$ for $(l, N)=1$, then $\mathbf{T}$ may be bigger than the classical Hecke algebra acting on the space $H^{0}\left(X_{1}(N), \omega \otimes \mathbf{C}\right)$ of classical modular forms of weight one. Let $G_{\mathbf{Q}}$ be the absolute Galois group of $\mathbf{Q}$. Let $G_{\mathbf{Q}, N}$ be the absolute Galois group of the maximal extension of $\mathbf{Q}$ unramified outside $N \infty$. Our main theorem is as follows:

Theorem 1.1. Let $\mathbf{T} \subset \operatorname{End}_{\mathcal{O}} H^{0}\left(X_{1}(N), \omega \otimes K / \mathcal{O}\right)$ denote the algebra generated by Hecke operators $T_{l}$ and $\langle l\rangle$ for all $l$ prime to $N$. There is a degree $d=2$ determinan 1 :

$$
\mathbf{D}: \mathbf{T}\left[G_{\mathbf{Q}}\right] \rightarrow \mathbf{T}, \quad P(\mathbf{D}, \sigma)=X^{2}-T(\sigma) X+D(\sigma),
$$

F.C. was supported in part by NSF Grant DMS-1701703.

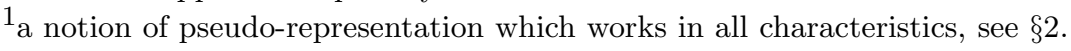


which is unramified outside $N \infty$ - equivalently, which factors through $\mathbf{T}\left[G_{\mathbf{Q}, N}\right]$ - such that for all primes $l \nmid N$, including $l=p$, one has

$$
T\left(\operatorname{Frob}_{l}\right)=T_{l} \text { and } D\left(\operatorname{Frob}_{l}\right)=\langle l\rangle .
$$

The ring $\mathbf{T}$ is a finite $\mathcal{O}$-algebra and is moreover a semi-local ring, and thus is a direct sum $\bigoplus \mathbf{T}_{\mathfrak{m}}$ of its completions at maximal ideals $\mathfrak{m}$. For each maximal ideal $\mathfrak{m}$ of $\mathbf{T}$, the residual determinant $\bar{P}: \mathcal{O}\left[G_{\mathbf{Q}}\right] \rightarrow \mathbf{T}_{\mathfrak{m}} / \mathfrak{m}=k$ arises from to a semi-simple Galois representation $\bar{\rho}$ over $\bar{k}$ (Theorem A of [Che14]). If this representation is irreducible, then $P$ itself also arises from a genuine representation, which, by a theorem of Carayol Car94, takes values in $\mathbf{T}_{\mathfrak{m}}$. It follows from Theorem 1.1 that the corresponding representation

$$
\rho: G_{\mathbf{Q}} \rightarrow \mathrm{GL}_{2}\left(\mathbf{T}_{\mathfrak{m}}\right)
$$

is unramified at $p$. For $p>2$, this is a consequence of Theorem 3.11 of [CG18]. Hence the main interest of this result is to residually reducible representations. However, the result is new even for absolutely irreducible representations when $p=2$ (although there are significant partial results by Wiese [Wie14]). Although the proof of Theorem 1.1] is similar to that of Theorem 3.11 of [CG18, it is more direct, and does not rely on any explicit analysis of the ordinary deformation rings of Snowden Sno18. Hence this paper can also be seen as providing a simplification of the proof of Theorem 3.11 of ibid. (See also Remark 3.3).

The existence of the determinant without any condition at $p$ is an easy consequence of the corresponding result in higher weight: first consider the action of $\mathbf{T}$ on $H^{0}\left(X_{1}(N), \omega / \varpi^{m}\right)$ and then multiply by a suitable power of the Hasse invariant which is Hecke equivariant. Hence the main content of this theorem is that the determinant is unramified at $p$.

\section{Determinants}

In this paper, we will use the term "pseudo-representation" as a catch-all to refer to various types of generalized representations. The first pseudo-representations were introduced by Wiles [Wil88] for 2-dimensional representations; these were later generalized to any dimension by Taylor [Tay91]. Following Roquier [Rou96], we will call Taylor-style pseudo-representations "pseudo-characters," because of their resemblance to the trace of a representation. In this paper, we will mainly consider the pseudo-representations of Chenevier Che14 called "determinants." These are more general and flexible than pseudo-characters, and in particular allow us to treat the case where $p=d=2$. We shall only be concerned with determinants of degree $d=2$.

We begin by recalling the notion of a determinant [Che14, pg. 223]. Let $G$ be a group and $A$ be a ring. Let $d$ be a positive integer. If $M$ is a free, rank- $d A$-module equipped with a linear $G$ action, then one may consider the family of characteristic polynomials associated to the elements of $A[G]$ acting on $M$. This family of polynomials is highly interdependent, and is a robust invariant of the representation $M$. Informally, a degree $d$ determinant is a pseudo-representation containing the information of a family of polynomials which satisfies the collection of common relations shared by all families of degree $d$ characteristic polynomials. If $B$ is an $A$-algebra, one can extend the action of $A[G]$ on $M$ to an action of $B[G]$ on $M \otimes_{A} B$, and also obtain corresponding characteristic polynomials over $B$ for elements in $B[G]$. Chenevier's definition of a determinant follows from the following two insights. First, the data of the characteristic polynomials for elements in $B[G]$ as one ranges over all $A$-algebras $B$ is equivalent to that of the literal determinants of the elements of $B[G]$ acting on $M \otimes_{A} B$ as 
one ranges over all $A$-algebras $B$ : the characteristic polynomial of an element $m \in B[G]$ is, by definition, the determinant of the endomorphism $X-m$ acting on $M \otimes_{A} B[X]$. Second, relations in families of characteristic polynomials arise via compatibilities of the determinant map. The literal determinants of the elements of $B[G]$ acting on $M \otimes_{A} B$ can be organized as a series of set theoretic maps det $: B[G] \rightarrow B$, one for each $A$-algebra $B$, which satisfy the following compatibilities:

(1) the maps det are natural in $B$,

(2) $\operatorname{det}(1)=1$ and the element $\operatorname{det}(x y)=\operatorname{det}(x) \operatorname{det}(y)$ for all $x, y \in B[G]$,

(3) and $\operatorname{det}(b x)=b^{d} \operatorname{det}(x)$, where $b \in B$ and $d$ is equal to the rank of $M$.

A determinant is simply a family of maps which are compatible in these three ways.

Definition 2.1. Let $A$ be a ring, $G$ be a topological group, and d be a positive integer. $A$ degree $d$ determinant is a continuous A-valued polynomial lau ${ }^{3} \mathbf{D}: A[G] \rightarrow A$, which is multiplicative and homogeneous of degree $d$. If $B$ is an A-algebra and $m \in B[G]$, we call $P(\mathbf{D}, m)(X):=\mathbf{D}(X-m) \in B[X]$ the characteristic polynomial of $m$.

Given a determinant $\mathbf{D}: A[G] \rightarrow A$ and an $A$-algebra $B$, the restriction of $\mathbf{D}$ to the category of $B$-algebras defines a determinant $\mathbf{D}_{B}: B[G] \rightarrow B$ on $B$. We call $\mathbf{D}_{B}$ the base change of $\mathbf{D}$ to $B$.

2.1. Determinants of degree $d=2$. Given a determinant $\mathbf{D}: A[G] \rightarrow A$ of degree 2 , the corresponding characteristic polynomials $P(\mathbf{D}, m) \in B[X]$ for $m \in B[G]$ have degree 2 and can be written in the form

$$
P(m)=P(\mathbf{D}, m)=X^{2}-T(m) X+D(m),
$$

for maps $T, D: B[G] \rightarrow B$. Note that the family of maps $D: B[G] \rightarrow B$ as $B$ ranges over all $A$-algebras is precisely the data which defines the polynomial law $\mathbf{D}$. In practice, our groups $G$ will always be Galois groups with the usual pro-finite topology, and our rings $A$ will either be $p$-adically complete semi-local $W(k)$-algebras with the $p$-adic topology or $p$-adic fields with the $p$-adic topology. We insist that all Galois representations and all determinants considered in this paper are continuous with respect to the topologies on $G$ and $A$.

In residue characteristic different from 2 and degree 2, one can recover $D$ from $T$ via the identity

$$
D(\sigma)=\frac{T(\sigma)^{2}-T\left(\sigma^{2}\right)}{2} .
$$

On the other hand, for any $p$, one can recover $T$ from $D$ by the formula

$$
T(\sigma)=D(\sigma+1)-D(\sigma)-1 \text {. }
$$

We have the following characterization of determinants of degree 2.

\footnotetext{
${ }^{2}$ All rings considered in this note will carry a Hausdorff topology, and, with the exception of group rings, will be commutative. Our terminology will suppress these topological and algebraic considerations. We use the terms module and algebra to denote a Hausdorff topological module and a commutative, Hausdorff topological algebra, respectively.

${ }^{3}$ An $A$-valued polynomial law between two $A$-modules $M$ and $N$ is by definition a natural transformation $N \otimes_{A} B \rightarrow M \otimes_{A} B$ on the category of commutative $A$-algebras $B$. A polynomial law is called multiplicative if $\mathbf{D}(1)=1$ and $\mathbf{D}(x y)=\mathbf{D}(x) \mathbf{D}(y)$ for all $x, y \in A[G] \otimes B$, and is called homogeneous of degree $d$, if $\mathbf{D}(x b)=b^{d} \mathbf{D}(x)$ for all $x \in A[G] \otimes B$ and $b \in B$. A polynomial law is called continuous if its characteristic polynomial map on $G$ given by $g \mapsto P(\mathbf{D}, g)$ is continuous.
} 
Lemma 2.2. Che14, Lemma 7.7] The set of determinants of $G$ over $A$ of degree 2 are in bijection with maps $(T, D)$ from $G$ to $A$ satisfying the following two conditions:

(1) $D: G \rightarrow A^{\times}$is a homomorphism,

(2) $T: G \rightarrow A$ is a function with $T(1)=2$, and such that, for all $g, h \in G$ :

(a) $T(g h)=T(h g)$,

(b) $D(g) T\left(g^{-1} h\right)-T(g) T(h)+T(g h)=0$.

In light of this lemma, we shall (from now on) regard a determinant $\mathbf{D}$ of $G$ over $A$ of degree 2 as precisely given by a pair of functions $(T, D)$ satisfying the equations above. Given $g \in G$, we have a corresponding characteristic polynomial $P(g)=X^{2}-T(g) X+D(g)$. By abuse of notation, we shall denote the pair $(T, D)$ by $P=(T, D)$. By [Che14, Lemma 7.7], the functions $T$ and $D$ extend to functions from $A[G]$ to $A$. In the case of $T$, this extension is the linear extension, and in the case of $D$, it can be constructed explicitly by using the equation for $D(x t+y s)$ given below. Note that $D$ as a function of $A[G]$ determines $T$ and hence $P$ and hence $\mathbf{D}$, but $D$ as a function of $G$ (in general) does not. Under this equivalence, the base change of a determinant $P:=(T, D)$ to an $A$-algebra $B$ corresponds to the determinant $f \circ P:=(f \circ T, f \circ D)$ obtained by post-composing the functions $T$ and $D$ with the structure homomorphism $f: A \rightarrow B$.

If $A$ is an algebraically closed field, then $(T, D)$ may be realized as the trace and (classical) determinant of an actual semisimple representation (Theorem A of [Che14]).

There is a well-defined notion of the kernel of $P$ (see [Che14, §1.4]), which in our case has the following simple description:

Lemma 2.3. The kernel of a determinant $P=(T, D)$ of degree 2 consist of the elements $x \in$ $A[G]$ satisfying the following two conditions:

(1) $T(x y)=0$ for all $y \in A[G]$,

(2) $D(x)=0$.

Proof. For polynomial laws of degree 2, we have (cf. Example 7.6 of [Che14])

$$
D(x t+y s)=D(x) t^{2}+(T(x) T(y)-T(x y)) t s+D(y) s^{2} .
$$

As follows from $\S 1.4$ of [Che14, we may compute the $x \in \operatorname{ker}(P)$ by finding the $x$ for which this expression is independent of $t$. Taking $y=1$ yields the equalities $T(x)=0$ and $D(x)=0$. Returning to the case of general $y$, we then deduce that $T(x y)=T(x) T(y)=0$.

Suppose that $H$ is a subgroup of $G$ such that $[h]-1 \in \operatorname{ker}(P)$ for all $h \in H$. In this case, by abuse of notation, we say that $\operatorname{ker}(P)$ contains $H$. If $\operatorname{ker}(P)$ contains $H$, then $\left[g h g^{-1}\right]-$ $1 \in \operatorname{ker}(P)$ for any $g \in G$, and (cf. Lemma 7.14 of Che14]) the determinant $P$ factors through $A[G / N]$, where $N$ is the normal closure of $H$. (That is, the functions $T$ and $D$ on $A[G]$ depend only on their image in the quotient $A[G / N]$.) In particular, to show that a determinant on $\mathcal{O}\left[G_{\mathbf{Q}}\right]$ is unramified at a prime $l$ (for example $l=p$ ), it suffices to show that the kernel contains some (any) choice of inertia subgroup $I_{l}$ at $l$, or equivalently:

Lemma 2.4. $I_{l}=H \subset G=G_{\mathbf{Q}}$ lies in the kernel of $P$ if and only if:

(1) $T(h g)=T(g)$ for all $h \in H=I_{l}$ and $g \in G=G_{\mathbf{Q}}$.

(2) $D(h-1)=0$ for all $h \in H=I_{l}$.

2.2. Ordinary Determinants. Let $\mathcal{O}$ be the ring of integers of a finite extension $\left[K: \mathbf{Q}_{p}\right]<$ $\infty$, let $\varpi$ be a uniformizer of $\mathcal{O}$, and suppose that $\mathcal{O} / \varpi=k$. Let $\bar{P}=(\bar{T}, \bar{D}): G_{\mathbf{Q}} \rightarrow k$ be a degree 2 determinant which is unramified outside $N p$. In practice, it will always be taken 
to be modular of level $\Gamma_{1}(N)$. Let us fix, once and for all, an embedding of $\overline{\mathbf{Q}}$ into $\overline{\mathbf{Q}}_{p}$, and hence inclusions:

$$
I_{p} \subset D_{p} \subset G_{\mathbf{Q}}
$$

where $I_{p}$ is the inertia group of $\mathbf{Q}_{p}$, and $D_{p}=\operatorname{Gal}\left(\overline{\mathbf{Q}}_{p} / \mathbf{Q}_{p}\right)$ is the decomposition group. Let us also fix a Frobenius element $\phi \in D_{p}$. There is a natural projection $D_{p} \rightarrow D_{p} / I_{p} \simeq \widehat{\mathbf{Z}}$ whose image is topologically generated by the image of $\phi$. Let $\epsilon: G_{\mathbf{Q}} \rightarrow \mathbf{Z}_{p}^{\times}$be the cyclotomic character; we may choose $\phi$ so that $\epsilon(\phi)=1$. Enlarging $k$ if necessary, let $\bar{\alpha}$ and $\bar{\beta}$ be the roots of the quadratic polynomial

$$
X^{2}-\bar{T}(\phi) X+\bar{D}(\phi)=0
$$

over $k$. We do not assume that these are necessarily distinct.

There are a number of slightly different definitions of ordinary Galois representations in the literature. Let us say that a 2-dimensional representation $\rho: G_{\mathbf{Q}_{p}} \rightarrow \mathrm{GL}_{2}\left(\overline{\mathbf{Q}}_{p}\right)$ is ordinary if the underlying 2-dimensional vector space $V$ admits a two step filtration $0 \subsetneq V^{\prime} \subsetneq V$ such that the action of $G_{\mathbf{Q}_{p}}$ on $V^{\prime \prime}=V / V^{\prime}$ is unramified. (This coincides, for example, with the definition of ordinary in SW97.) We furthermore say that $\rho$ is ordinary of weight $n$ if the action of $G_{\mathbf{Q}_{p}}$ on $V^{\prime}$ is via an unramified twist of $\epsilon^{n-1}$. By abuse of notation, if $\rho$ : $G_{\mathbf{Q}} \rightarrow \mathrm{GL}_{2}\left(\overline{\mathbf{Q}}_{p}\right)$ is a global Galois representation, we say that it is ordinary if $\left.\rho\right|_{G_{\mathbf{Q}_{p}}}$ is ordinary (respectively, ordinary of weight $n$ ). When a representation is ordinary, various relations are imposed on its associated determinant. We collect several of these relations common to all ordinary 2-dimensional representations of weight $n$, and then define that a determinant $P=(T, D): A\left[G_{\mathbf{Q}}\right] \rightarrow A$ of degree 2 to be an "ordinary determinant of weight $n$ " if and only if it satisfies these conditions. Our definition includes the auxiliary data of an "eigenvalue" $\alpha \in A^{\times}$of the Frobenius element $\phi$. This "eigenvalue" satisfies some relations shared by every value which occurs as the eigenvalue of $\phi$ on a choice of unramified quotient of $\left.\rho\right|_{G_{\mathbf{Q}_{p}}}$ in an 2-dimensional ordinary representation of weight $n$. We will be interested in deformations of $\bar{P}$ to Artinian local rings $(A, \mathfrak{m})$ which are ordinary of weight $n$.

Definition 2.5. Let $(A, \mathfrak{m})$ be a Noetherian local ring with residue field $k$. An ordinary determinant $P: A\left[G_{\mathbf{Q}}\right] \rightarrow A$ of degree 2 and weight $n$ with eigenvalue $\alpha \in A^{\times}$consists of a pair $(P, \alpha)$ where $P=(T, D): A\left[G_{\mathbf{Q}}\right] \rightarrow A$ is a degree $d=2$ determinant satisfying the following properties:

(1) $P(h)=(X-1)(X-\psi(h))$ for all $h \in I_{p}$, where $\psi=\epsilon^{n-1}$.

(2) $\alpha$ is a root of $X^{2}-T(\phi) X+D(\phi)$.

(3) For all $h \in I_{p},(h-\psi(h))(\phi-\alpha) \in \operatorname{ker}(P)$. Equivalently, for all $g \in G_{\mathbf{Q}}$ and $h \in I_{p}$,

$$
T(g(h-\psi(h))(\phi-\alpha))=T(g h \phi)-\psi(h) T(g \phi)-T(g h) \alpha+T(g) \psi(h) \alpha=0 .
$$

The first two conditions of this definition are self-explanatory. The last may be somewhat surprising to the reader; note that it involves a condition on general elements $g \in G_{\mathbf{Q}}$ rather than simply being a condition on the decomposition group. This turns out to be necessary, because the determinant (or pseudo-character) associated to the decomposition group of a locally reducible representation does not know which character comes from the quotient and which comes from the submodule. The idea behind this definition, as we shall see shortly below, is to capture the notion that the product $(h-\psi(h))(\phi-\alpha)$ is identically zero, rather than just of the form $\left(\begin{array}{ll}0 & * \\ 0 & 0\end{array}\right)$. There is presumably a close relationship between this definition 
and the definition of ordinary pseudo-characters in Wake, Wang-Erickson (see [WWE17] and $\S 7.3$ of [WE18]), although in our context it is important that we can work in non- $p$ distinguished situations by choosing an eigenvalue of Frobenius, which amounts to a partial resolution of the corresponding deformation rings (presumably such modifications could also be adapted to [WE18]). On the other hand, we do exploit the crucial idea due to WangErickson that the notion of ordinarity for pseudo-representations should be a global rather than local condition. The following lemma provides a justification for the final condition above, and the proof provides a motivation for its definition.

Lemma 2.6. Suppose that $f$ is a classical modular eigenform of level $\Gamma_{0}(p) \cap \Gamma_{1}(N)$ with Nebentypus character $\chi$ of weight $n \geq 2$ with coefficients in $\mathcal{O}$, and suppose that $\alpha$ is the $U_{p^{-}}$ eigenvalue of $f$. Assume that $f$ is ordinary (equivalently, that $\alpha$ has trivial valuation). Then the associated determinant $P_{f}: \mathcal{O}\left[G_{\mathbf{Q}}\right] \rightarrow \mathcal{O}$ is ordinary with eigenvalue $\alpha$, weight $n$, and is unramified outside $N p$.

Note that $f$ in Lemma 2.6 need not be new at either the prime $p$ or primes dividing $N$.

Proof. Since $\mathcal{O}$ has characteristic zero, there is a Galois representation (via [Del71])

$$
\rho_{f}: G_{\mathbf{Q}, N p} \rightarrow \mathrm{GL}_{2}\left(\overline{\mathbf{Q}}_{p}\right)
$$

associated to $f$. The determinant $P_{f}=\left(T_{f}, D_{f}\right)$ is (by definition) the determinant associated to the representation $\rho_{f}$. Since $\rho_{f}$ factors through $G_{\mathbf{Q}, N p}$, this determinant is unramified at primes outside $N p$. Let $\lambda_{\alpha}: G_{\mathbf{Q}_{p}} \rightarrow \overline{\mathbf{Q}}_{p}^{\times}$denote the unramified character which sends Frob ${ }_{p}$ to $\alpha$. We collect the following facts concerning the Galois representation $\rho_{f}$ :

Fact 2.7. The representation $\rho_{f}$ has the following properties 4

(1) The representation $\rho_{f}$ is unramified outside $N p$. The trace and (classical) determinant of $\rho_{f}\left(\mathrm{Frob}_{l}\right)$ are equal to $a_{l}(f)$ and $l^{n-1} \chi(l)$ respectively. The (classical) determinant of $\rho_{f}$ is the character $\chi \epsilon^{n-1}$, where $\chi$ is unramified outside $N$.

(2) If $f$ is old at level $p$, and the corresponding eigenform $g$ of level $\Gamma_{1}(N)$ has $T_{p}$ eigenvalue $a_{p}$, then $\alpha$ is the unit root of $X^{2}-a_{p} X+p^{n-1} \chi(p)$, and

$$
\left.\rho_{f}\right|_{D_{p}}=\left.\rho_{g}\right|_{D_{p}} \sim\left(\begin{array}{cc}
\epsilon^{n-1} \lambda_{\alpha}^{-1} \chi & * \\
0 & \lambda_{\alpha}
\end{array}\right) .
$$

(3) If $f$ is new at level $p$, then $n=2$,

$$
\left.\rho_{f}\right|_{D_{p}} \sim\left(\begin{array}{cc}
\epsilon \lambda_{\alpha} & * \\
0 & \lambda_{\alpha}
\end{array}\right)
$$

and $\left.\chi\right|_{D_{p}} \simeq \lambda_{\alpha}^{2}$.

\footnotetext{
${ }^{4}$ Some References: The fact that $\rho_{f}$ is unramified outside $N p$ already follows from the original construction of Deligne [Del71]. Since the Nebentypus character has conductor dividing $N$, the corresponding Galois representation $\chi$ is certainly unramified outside $N$. The second claim follows immediately from Wil88, Theorem 2]. Consider the third claim, so we are assuming that $f$ is new at $p$. If one writes $\chi_{0}=\chi_{p} \chi_{N}$ where $\chi_{p}$ and $\chi_{N}$ are characters corresponding to the identification $(\mathbf{Z} / N p \mathbf{Z})^{\times}=(\mathbf{Z} / p \mathbf{Z})^{\times} \oplus(\mathbf{Z} / N \mathbf{Z})^{\times}$, then (by assumption) $\chi_{p}$ is trivial. It follows (see $\S 1$ of AL78) that $f$ is an eigenform for operator $W_{p}$ with eigenvalue $\lambda_{p}(f)$ satisfying $\lambda_{p}^{2}(f)=\chi(p)$ (AL78, Proposition 1.1]). On the other hand, by AL78, Theorem 2.1], we deduce that $\alpha^{2}=\lambda_{p}^{2}(f) p^{n-2}=\chi(p) p^{n-2}$. Under our assumption that $\alpha$ is a $p$-adic unit, this can only occur when the weight $n=2$. When $n=2$, however, we can appeal to DDT97, Theorem 3.1(e)] which gives a detailed description of the local properties of Galois representations associated to ordinary forms. Finally, the identification of $\left.\chi\right|_{D_{p}}$ with $\lambda_{\alpha}^{2}$ follows either by considering determinants or the identity $\alpha^{2}=\chi(p)$ discussed above.
} 
Using these properties, we see that the required conditions for $P_{f}$ to be ordinary with eigenvalue $\alpha$ are easily met with the possible exception of the final condition. For this, note that from the explicit descriptions above there exists a basis such that:

$$
\left.\rho_{f}\right|_{I_{p}}=\left(\begin{array}{cc}
\psi & * \\
0 & 1
\end{array}\right), \quad \rho_{f}(\phi)=\left(\begin{array}{cc}
\chi(\phi) \alpha^{-1} & * \\
0 & \alpha
\end{array}\right),
$$

where $\operatorname{det}\left(\rho_{f}\right)=\epsilon^{n-1} \chi$. We find, with $h \in I_{p}$, that, in $M_{2}(\mathcal{O})$,

$$
\left(\rho_{f}(h)-\psi(h)\right)\left(\rho_{f}(\phi)-\alpha\right)=\left(\begin{array}{ll}
0 & 0 \\
0 & 0
\end{array}\right) .
$$

It follows that

$$
T_{f}(s(h-\psi(h))(\phi-\alpha))=\operatorname{tr}\left(\rho_{f}(s)\left(\rho_{f}(h)-\psi(h)\right)\left(\rho_{f}(\phi)-\alpha\right)\right)=0
$$

for all $s \in G_{\mathbf{Q}}$.

We now fix our choice of $\bar{P}$. Let $\bar{P}: k\left[G_{\mathbf{Q}}\right] \rightarrow k$ be the determinant associated to a mod $\varpi$ weight one eigenform $g$ of level $\Gamma_{1}(N)$, i.e. the determinant associated to the Galois representation classically attached to $g$ [Gro90, Proposition 11.1]. Suppose that $g$ has Nebentypus character $\chi$ and $T_{p}$-eigenvalue $a_{p}$, and let $\bar{\alpha}$ and $\bar{\beta}$ be the roots of $X^{2}-a_{p} X+\chi(p)$, which we assume (enlarging $\mathcal{O}$ if necessary) are $k$-rational.

Lemma 2.8. Let $n \equiv 1 \bmod (p-1)$ be an integer. The determinant $\bar{P}$ is ordinary of degree 2 and weight $n$ with eigenvalue $\bar{\alpha}$ and is unramified outside $N p$. If $n>1$, there is an eigenform $f$ of level $\Gamma_{1}(N) \cap \Gamma_{0}(p)$ and weight $n$ which is ordinary at $p$ for which the $U_{p}$-eigenvalue of $f$ is congruent to $\bar{\alpha} \bmod \varpi$ such that $\bar{P}=\bar{P}_{f}$.

By symmetry, the result holds with $\bar{\alpha}$ replaced by $\bar{\beta}$.

Proof. Since $\epsilon^{n-1}$ is trivial $\bmod \varpi$ for $n \equiv 1 \bmod (p-1)$, if $\bar{P}$ is ordinary with eigenvalue $\bar{\alpha}$ for one such $n$, it is ordinary for all such $n$. Suppose we can construct an eigenform $h$ modulo $\varpi$ of level $\Gamma_{1}(N)$ of weight $p$ and such that the $T_{p}$-eigenvalue of $h$ is congruent to $\bar{\alpha}$ $\bmod \varpi$, and such that $\bar{P}_{h}=\bar{P}$. By multiplying by powers of the Hasse invariant, we deduce that there also exists such a form in any weight $n \equiv 1 \bmod (p-1)$ such that $n>1$. All mod $\varpi$ modular forms in weights $n>1$ and level $\Gamma_{1}(N)$ lift to characteristic zero. (This follows as in [Kat73, Theorem 1.7.1], the running assumption that $N \geq 5$ guaranteeing that $X_{1}(N)$ is a fine moduli space.) Moreover, using the Deligne-Serre lifting lemma ([DS74, Lemme 6.11]), one can always choose a lift $h$ which is an eigenform for all the Hecke operators. The lifted form $h$ of weight $\Gamma_{1}(N)$ has weight $n>1$ and $T_{p}$-eigenvalue $\bar{\alpha} \bmod \varpi$. But now the ordinary stabilization $f$ of $h$ of level $\Gamma_{1}(N) \cap \Gamma_{0}(p)$ has has $U_{p}$-eigenvalue $\bar{\alpha} \bmod \varpi$, and $\bar{P}_{f}=\bar{P}_{h}=\bar{P}$, as required. Finally, we deduce from Lemma 2.6 applied to $f$ that $\bar{P}$ is ordinary with eigenvalue $\bar{\alpha}$ (of weight $n$ and unramified outside $N p$ ). Thus it remains to construct $h$ from $g$.

If $A$ is the Hasse invariant, then $A g$ is a modular form mod $\varpi$ of level $\Gamma_{1}(N)$ and weight $p$ which is an eigenform for all Hecke operators except for $T_{p}$, and moreover has the same eigenvalues as $g$. The same is true of $T_{p}(A g)$ and also $A\left(T_{p} g\right)$ (the latter is just $a_{p} A g$ ). On the level of $q$-expansions, there are equalities $A g=g$ and $A T_{p}(g)-T_{p}(A g)=V g$ respectively. Hence $h=g-\bar{\beta} V g$ is a weight $p$ modular eigenform $\bmod \varpi$ of level $\Gamma_{1}(N)$ with $\bar{P}_{f}=\bar{P}$ 
and with $T_{p}$-eigenvalue $\bar{\alpha}$. To see that $T_{p} h=\bar{\alpha} h$, note (cf. Gro90, §4], especially (4.7)) that $T_{p}(V g)=A g$ and $T_{p} A g=a_{p} A g-\chi(p) V g$, and hence

$$
\begin{aligned}
T_{p} h & =T_{p}(A g-\bar{\beta} V g) \\
& =a_{p} A g-\chi(p) V g-\bar{\beta} A g \\
& =(\bar{\alpha}+\bar{\beta}) A g-\bar{\alpha} \bar{\beta} V g-\bar{\beta} A g \\
& =\bar{\alpha}(A g-\bar{\beta} V g)=\bar{\alpha} h .
\end{aligned}
$$

Let $R=R^{\text {univ }}$ denote the universal deformation ring of $\bar{P}$ (cf. [Che14, Proposition 7.59]) unramified outside $N p$. It pro-represents the functor which, for Artinian local $W(k)$-algebras $(A, \mathfrak{m})$ with residue field $A / \mathfrak{m}=k$, consists of determinants $P=(T, D)$ valued in $A$ whose mod $\mathfrak{m}$ reduction is $\bar{P}$. Let $P^{\text {univ }}=\left(T^{\text {univ }}, D^{\text {univ }}\right)$ denote the corresponding universal determinant. We define a mild variant on this ring by considering such determinants: $P=(T, D): A\left[G_{\mathbf{Q}}\right] \rightarrow A$ together with a root $\alpha$ of $X^{2}-T(\phi) X+D(\phi)$. The result is an extension $\widetilde{R}$ of $R$ given by

$$
\widetilde{R}=R[\alpha] /\left(\alpha^{2}-T^{\text {univ }}(\phi) \alpha+D^{\text {univ }}(\phi)\right) .
$$

The ring $R$ is a local $W(k)$-algebra, but the ring $\widetilde{R}$ is a semi-local $W(k)$-algebra with either one or two maximal ideals. It has 2 maximal ideals precisely when the polynomial $\alpha^{2}-$ $\bar{T}(\phi) \alpha+\bar{D}(\phi) \in k[\alpha]$ is separable.

Definition 2.9. Let $\widetilde{D}_{n}^{\dagger}(A)$ denote the functor which, for Artinian local rings $(A, \mathfrak{m})$ with residue field $A / \mathfrak{m}=k$, consists of ordinary determinants $\left(P, \alpha_{0}\right)$ of weight $n$ unramified outside $N p$, where $P$ is a deformation of $\bar{P}$ to $A$, and $n \equiv 1 \bmod (p-1)$ is a positive integer.

Note that elements in $\widetilde{D}_{n}^{\dagger}(k)$ are in bijection with choices of $\bar{\alpha} \in k$ so that $\bar{P}$ is ordinary of weight $n$ with eigenvalue $\bar{\alpha}$. By Lemma 2.8 , such a choice of eigenvalue exists. Furthermore since $\bar{\alpha}$ is a root $X^{2}-\bar{T}(\phi) X+\bar{D}(\phi)$, the size of $\widetilde{D}_{n}^{\dagger}(k)$ is at most 2. For each root $\bar{\alpha} \in$ $k$ of $X^{2}-\bar{T}(\phi) X+\bar{D}(\phi)$, consider the sub-functor $\widetilde{D}_{n}^{\dagger, \bar{\alpha}}(A) \subseteq \widetilde{D}_{n}^{\dagger}(A)$ consisting of pairs with $\left(P, \alpha_{0}\right)$ such that $\alpha_{0} \equiv \bar{\alpha} \bmod \mathfrak{m}$. The functor $\widetilde{D}_{n}^{\dagger}$ decomposes as the coproduct

$$
\widetilde{D}_{n}^{\dagger}(A)=\coprod_{(\bar{P}, \bar{\alpha}) \in \widetilde{D}_{n}^{\dagger}(k)} \widetilde{D}_{n}^{\dagger, \bar{\alpha}}(A),
$$

and each of the sub-functors $\widetilde{D}_{n}^{\dagger, \bar{\alpha}}$ are pro-represented by a (potentially trivial) Noetherian local $W(k)$-algebra $\widetilde{R}_{n}^{\dagger, \bar{\alpha}}$. By abuse of terminology, we will say $\widetilde{D}_{n}^{\dagger}$ is pro-represented by the semi-local ring

$$
\widetilde{R}_{n}^{\dagger}:=\bigoplus_{(\bar{P}, \bar{\alpha}) \in \widetilde{D}_{n}^{\dagger}(k)} \widetilde{R}_{n}^{\dagger, \bar{\alpha}} .
$$

Explicitly, if $\widetilde{P}^{\text {univ }}$ is the base change of $P^{\text {univ }}$ to the $R$-algebra $\widetilde{R}$, then $\widetilde{R}_{n}^{\dagger}$ is the quotient of $\widetilde{R}$ by the ideal generated by all the relations which obstruct $P^{\text {univ }}$ from being ordinary of weight $n$ with eigenvalue $\alpha$. The universal determinant $P_{n}^{\dagger, \text { univ }}$ is base change of $\widetilde{P}^{\text {univ }}$ to $\widetilde{R}_{n}^{\dagger}$ and the universal eigenvalue is $\alpha$.

The determinant $P^{\dagger}$,univ itself is valued in the subring $R_{n}^{\dagger}$ of $\widetilde{R}_{n}^{\dagger}$, which is the image of $R \subset \widetilde{R}$. However, the element $\alpha$ will not, in general, lie in $R_{n}^{\dagger}$. The extra data of $\alpha$ records, implicitly, the "choice" of realizing the corresponding determinant as ordinary. (The 
same determinant $P$ can in principle be realized as an ordinary determinant $(P, \alpha)$ for different values of $\alpha$.)

The following result is the key proposition which allows us to prove that certain ordinary determinants are unramified. The idea is that, given a representation which is ordinary, the more the representation is ramified, the more the choice of ordinary eigenvalue $\alpha$ is pinned down by the Galois representation, because the ramification structure gives a partial filtration on the representation which mirrors the ordinary filtration. The extreme case, in which $\alpha$ cannot be distinguished from the other root $\alpha^{-1} D(\phi)$ of the characteristic polynomial of $\phi$, should only occur when the representation is unramified. While these claims are obvious for $\overline{\mathbf{Q}}_{p}$-valued representations, the key property of our definition is that one can prove this for any quotient of $\widetilde{R}_{n}^{\dagger}$.

Proposition 2.10. Let $\widetilde{R}_{n}^{\dagger} \rightarrow \widetilde{S}$ be a surjective homomorphism of $W(k)$-algebras, and let $S$ denote the image of $R_{n}^{\dagger}$ in $\widetilde{S}$. Suppose that $\widetilde{S} / S$ is a free $S$-module of rank one, or equivalently, that the annihilator of $\widetilde{S} / S$ as an $S$-module is trivial. Then the corresponding determinant $P$ valued in $S$ is unramified.

Proof. We first verify that $D(h-1)=0$ without any assumptions. From first condition of Definition 2.5 we see that $D(h)=\psi(h)$ and $T(h)=1+\psi(h)$, and thus, from Equation (11) in the proof of Lemma 2.3, we deduce that

$$
D(h-1)=D(h)-(T(h) T(1)-T(h))+D(1)=\psi(h)-(\psi(h)+1)+1=0 .
$$

We now turn to the second condition of Lemma 2.4. The module $\widetilde{S} / S$ is a cyclic $S$-module generated by $\alpha$, so it is free if and only if the annihilator of $\alpha$ is trivial. We have by definition the identity (for $s \in G_{\mathbf{Q}}$ and $h \in I_{p}$ and $\psi=\epsilon^{n-1}$ )

$$
T(\operatorname{sh} \phi)-\psi(h) T(s \phi)-T(s h) \alpha+T(s) \psi(h) \alpha=0 .
$$

We may re-arrange this to obtain the identity:

$$
\alpha(T(s h)-T(s) \psi(h))=T(s h \phi)-\psi(h) T(s \phi) .
$$

Note that the value $T(s)$ for any $s \in G_{\mathbf{Q}}$ lands in $S$, as does the image of any element of $W(k)$, and hence it follows that

$$
\alpha(T(s h)-T(s) \psi(h))=0 \in \widetilde{S} / S .
$$

Take $g$ to be the identity, so $T(s h)=T(h)=1+\psi(h)$ and $T(s)=2$. Then we deduce that

$$
\alpha(1-\psi(h))=0 \in \widetilde{S} / S
$$

for all $h \in I_{p}$. If $\widetilde{S} / S$ is free, then its annihilator of $\alpha$ is trivial, and thus $\psi(h)=1$ for all $h$. But we then deduce for the same reason that $T(s h)-T(s) \psi(h)=T(s h)-T(s)=0$ for all $s \in G_{\mathbf{Q}}$ and $h \in I_{p}$, from which it follows by Lemma 2.4 (note that $T(s h)=T(h s)$ ) that $I_{p}$ is contained in the kernel.

\section{Galois Deformations}

By Lemma 2.8, our fixed determinant $\bar{P}=(\bar{T}, \bar{D}): k\left[G_{\mathbf{Q}}\right] \rightarrow k$ is associated to an ordinary $\bmod \varpi$ eigenform of level $\Gamma_{1}(N) \cap \Gamma_{0}(p)$ in each weight $n \geq 2$ satisfying $n \equiv 1 \bmod p-1$. Given our choice of Frobenius element $\phi \in D_{p} \subset G_{\mathbf{Q}}$, recall that $\bar{\alpha}$ and $\bar{\beta}$ are the roots of the polynomial

$$
\bar{P}(\phi)=X^{2}-\bar{T}(\phi) X+\bar{D}(\phi)
$$

We start by considering determinants arising from forms of higher weight. 
Lemma 3.1. Let $n \geq 2$ be an integer such that $n \equiv 1 \bmod p-1$. Let $\widetilde{\mathbf{T}}_{n}$ denote the $\mathcal{O}$-algebra of endomorphisms of

$$
M_{n}\left(\Gamma_{0}(p) \cap \Gamma_{1}(N), \mathcal{O}\right)
$$

generated by Hecke operators $T_{l}$ and $\langle l\rangle$ for $l$ prime to $N p$, together with $U_{p}$. Let $\mathfrak{m}$ denote the ideal of $\widetilde{\mathbf{T}}_{n}$ generated by $\varpi$ and by any lift in $\widetilde{\mathbf{T}}_{n}$ of the following elements of $\widetilde{\mathbf{T}}_{n} / \varpi$ : the operators $T_{l}-\bar{T}\left(\right.$ Frob $\left._{l}\right)$ and $\langle l\rangle l^{n-1}-\bar{D}\left(\operatorname{Frob}_{l}\right)$ for $(l, N p)=1$, and $\left(U_{p}-\bar{\alpha}\right)\left(U_{p}-\bar{\beta}\right)$. Assume that $\bar{P}$ is associated to an ordinary mod $\varpi$ eigenform of level $\Gamma_{1}(N) \cap \Gamma_{0}(p)$ and weight $n$ with $U_{p}$-eigenvalue congruent to either $\bar{\alpha}$ or $\bar{\beta}$ modulo $\varpi$, so that $\mathfrak{m}$ is a proper ideal. Then there exists a canonical surjection of semi-local rings

$$
\widetilde{R}_{n}^{\dagger} \rightarrow \widetilde{\mathbf{T}}_{n, \mathfrak{m}}
$$

sending $\alpha \in \widetilde{R}_{n}^{\dagger}$ to $U_{p}$.

Remark 3.2. If $\mathbf{T}_{n} \subset \widetilde{\mathbf{T}}_{n}$ denotes the subring generated by the all the Hecke operators except $U_{p}$, then $\mathfrak{m} \cap \mathbf{T}_{n}$ is maximal. However, $\mathfrak{m}$ itself need not be maximal. Throughout the rest of the paper, we let $\widetilde{\mathbf{T}}_{n, \mathfrak{m}}$ denote the completion $\widetilde{\mathbf{T}}_{n, \mathfrak{m}}:=\operatorname{proj} \lim \widetilde{\mathbf{T}}_{n} / \mathfrak{m}^{r}$ - it need not be a local ring. The Hecke algebra $\widetilde{\mathbf{T}}_{n, \mathfrak{m}}$ is non-local precisely when $\bar{\alpha} \neq \bar{\beta}$ and when $\bar{P}$ is associated to an ordinary mod $\varpi$ eigenform with $U_{p}$-eigenvalue congruent to $\bar{\alpha} \bmod \varpi$ and is also associated to an eigenform with $U_{p}$-eigenvalue congruent to $\bar{\beta} \bmod \varpi$. In that case, the ideals $\mathfrak{m}_{\bar{\alpha}}$ and $\mathfrak{m}_{\bar{\beta}}$ obtained by adjoining any lift of $U_{p}-\bar{\alpha}$ or $U_{p}-\bar{\beta}$ respectively from $\widetilde{\mathbf{T}}_{n} / \varpi$ to $\mathfrak{m}$ are both maximal, and there is an isomorphism $\widetilde{\mathbf{T}}_{n, \mathfrak{m}} \cong \widetilde{\mathbf{T}}_{n, \mathfrak{m}_{\bar{\alpha}}} \oplus \widetilde{\mathbf{T}}_{n, \mathfrak{m}_{\bar{\beta}}}$. Working with semi-local rings allows us to treat the cases $\bar{\alpha}=\bar{\beta}$ and $\bar{\alpha} \neq \bar{\beta}$ simultaneously. If $M$ is a module for $\widetilde{\mathbf{T}}_{n}$, then, when $\mathfrak{m}$ is not maximal, there is also a corresponding identification $M_{\mathfrak{m}}:=$ $\operatorname{proj} \lim M / \mathfrak{m}^{r}=M_{\mathfrak{m}_{\bar{\alpha}}} \oplus M_{\mathfrak{m}_{\bar{\beta}}}$.

Proof of Lemma 3.1. Consider an embedding $K \rightarrow L$, where $L$ is a field which contains the eigenvalues of all elements of $\widetilde{\mathbf{T}}_{n}$. The Hecke algebra $\widetilde{\mathbf{T}}_{n}$ acts faithfully on $M_{n}\left(\Gamma_{0}(p) \cap\right.$ $\left.\Gamma_{1}(N), L\right)$. Recall that $\mathbf{T}_{n} \subset \widetilde{\mathbf{T}}_{n}$ denotes the subring generated by Hecke operators away from $N p$ (i.e. without $\left.U_{p}\right)$. For each newform $h$ which contributes to $M_{n}\left(\Gamma_{0}(p) \cap \Gamma_{1}(N), L\right)$, there is a corresponding vector space $V(h) \subset M_{n}\left(\Gamma_{0}(p) \cap \Gamma_{1}(N), L\right)$ generated by $h$ together with the oldforms associated to $h$. (The space $V(h)$ can also be identified with the invariants $\pi^{\Gamma_{1}(N) \cap \Gamma_{0}(p)}$, where $\pi$ is the smooth admissible $\mathrm{GL}_{2}\left(\mathbf{A}^{(\infty)}\right)$-representation over $L$ generated by $h$.) There is a $\mathbf{T}_{n}$-equivariant isomorphism

$$
M_{n}\left(\Gamma_{0}(p) \cap \Gamma_{1}(N), L\right) \simeq \bigoplus_{g} V(h),
$$

where $\mathbf{T}_{n}$ acts on $V(h)$ through scalars corresponding to the homomorphism $\eta_{h}: \mathbf{T}_{n} \rightarrow L$ sending $T_{l}$ to $a_{l}(h)$ and $\langle l\rangle$ to $l^{n-1} \chi(l)$ where $\chi$ is the Nebentypus character of $h$. Let us now consider the action of the operator $U_{p}$. For each map $\eta_{h}: \mathbf{T}_{n} \rightarrow L$ (which corresponds to a fixed Galois representation $\rho_{h}$ ) one of the following two things happens:

(1) The newform $h$ has level $\Gamma_{0}(p)$ at $p$, in which case $U_{p}$ acts on $V(h)$ via a scalar.

(2) The newform $h$ has level $\Gamma_{0}(1)$ at $p$, in which case $U_{p}$ acts on $V(h)$ and satisfies the identity $U_{p}^{2}-a_{p} U_{p}+p^{n-1} \chi(p)=0$.

In particular, the algebra $\widetilde{\mathbf{T}}_{n}$ will always acts semi-simply in the first case and act semisimply in the second case as long as the corresponding polynomial $X^{2}-a_{p} X+p^{n-1} \chi(p)$ has 
distinct roots. This is known in general only under the assumption of the Tate conjecture (cf. CE98), but it can certainly only fail to happen when $a_{p}^{2}=4 p^{n-1} \chi(p)$, which would force the (multiple) eigenvalue of $U_{p}$ to have positive valuation (since $n \geq 2$ ). In particular, such forms do not contribute to $M_{n}\left(\Gamma_{0}(p) \cap \Gamma_{1}(N), \mathcal{O}\right)_{\mathfrak{m}} \otimes_{\mathcal{O}} L$, because (since $\mathfrak{m}$ contains the preimage of $\left(U_{p}-\bar{\alpha}\right)\left(U_{p}-\bar{\beta}\right)$ for non-zero $\bar{\alpha}$ and $\left.\bar{\beta}\right)$ the element $U_{p}$ acts invertibly on this space. (Recall, following Remark 3.2, that when $\mathfrak{m}$ is contained in two primes, $M_{n}\left(\Gamma_{0}(p) \cap \Gamma_{1}(N), \mathcal{O}\right)_{\mathfrak{m}}$ is simply the direct sum of $M_{n}\left(\Gamma_{0}(p) \cap \Gamma_{1}(N), \mathcal{O}\right)_{\mathfrak{m}_{\bar{\alpha}}}$ and $\left.M_{n}\left(\Gamma_{0}(p) \cap \Gamma_{1}(N), \mathcal{O}\right)_{\mathfrak{m}_{\bar{\beta}}}.\right)$ It follows that there is an injection

$$
i_{n}: \widetilde{\mathbf{T}}_{n, \mathfrak{m}} \hookrightarrow \bigoplus_{f} L,
$$

where the sum ranges over all $\widetilde{\mathbf{T}}_{n}$-eigenforms $f \in M_{n}\left(\Gamma_{0}(p) \cap \Gamma_{1}(N), L\right)$ such that $\bar{P}_{f}=$ $\bar{P}$ and the $U_{p}$-eigenvalue is congruent either to $\bar{\alpha}$ or $\bar{\beta}$. We identify $\widetilde{\mathbf{T}}_{n, \mathfrak{m}}$ with its image under $i_{n}$. For each of the forms $f$ above, denote the $U_{p}$-eigenvalue by $\alpha(f)$. By Lemma 2.6, the determinants $P_{f}$ are ordinary with eigenvalues $\alpha(f)$, weight $n$, and unramified outside $N p$. Hence, for each form $f$ there is a homomorphism

$$
i_{f}: \widetilde{R}_{n}^{\dagger} \rightarrow L
$$

such that $i_{f} \circ P^{\dagger, \text { univ }}=P_{f}$ and which maps $\alpha$ to $\alpha(f)$. Taking the direct sum of the maps $i_{f}$, we obtain a homomorphism

$$
j_{n}: \widetilde{R}_{n}^{\dagger} \rightarrow \bigoplus_{f} L
$$

under which $\alpha$ maps to $U_{p}, T\left(\right.$ Frob $\left._{l}\right)$ maps to $T_{l}$, and $D\left(\right.$ Frob $\left._{l}\right)$ maps to $\langle l\rangle$. We conclude that $j_{n}$ factors through a surjective homomorphism

$$
\widetilde{R}_{n}^{\dagger} \rightarrow \widetilde{\mathbf{T}}_{n, \mathfrak{m}}
$$

under which $\alpha$ maps to $U_{p}$.

We are now ready to prove the main theorem.

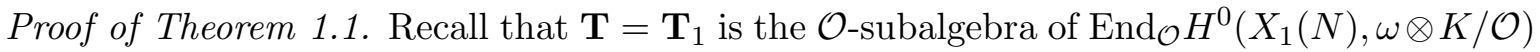
generated by $T_{l}$ and $\langle l\rangle$ for $(l, N)=1$. This ring contains $T_{p}$, but the element $T_{p}$ in weight one is also generated by the other Hecke operators (see, for example, Lemma 3.1 of [Cal18]). For each positive integer $m$, let $\mathbf{T}(m)$ denote the image $\mathbf{T}$ in End $\mathcal{O} H^{0}\left(X_{1}(N), \omega / \varpi^{m}\right)$. The ring $\mathbf{T} \cong \lim \mathbf{T}(m)$. Therefore, to prove Theorem 1.1, it suffices to construct for each $m>0$ a degree $d=2$ determinant

$$
\mathbf{D}_{m}: \mathbf{T}(m)\left[G_{\mathbf{Q}}\right] \rightarrow \mathbf{T}(m), \quad P\left(\mathbf{D}_{m}, \sigma\right)=X^{2}-T_{m}(\sigma) X+D_{m}(\sigma),
$$

which is unramified outside $N \infty$, and such that for all primes $l \nmid N$ (including $l=p$ ) the characteristic polynomial of $\mathrm{Frob}_{l}$ satisfies

$$
T_{m}\left(\text { Frob }_{l}\right)=T_{l} \text { and } D_{m}\left(\text { Frob }_{l}\right)=\langle l\rangle .
$$

In the remainder of the proof, we will assume that $m>0$ is fixed, and will denote by an abuse of notation $\mathbf{T}(m)$ by $\mathbf{T}$.

There is a decomposition $\mathbf{T}=\bigoplus \mathbf{T}_{\mathfrak{m}}$ over the maximal ideals $\mathfrak{m}$ of $\mathbf{T}$. Hence, it suffices to construct the desired determinant after completing at a maximal ideal $\mathfrak{m}$ of $\mathbf{T}$. Let $\bar{P}$ denote our fixed modular residual determinant, which we have assumed is supported in weight one, and let $\mathfrak{m}$ denote the maximal ideal which is the kernel of the corresponding map $\mathbf{T} \rightarrow k$. 
Let $\widetilde{\mathbf{T}}_{n}$ denote the Hecke algebra of Lemma 3.1 in weight $n:=1+p^{m-1}(p-1)$ which contains $U_{p}$ (and has coefficients in $\mathcal{O}$ ). By abuse of notation, we also let $\mathfrak{m}$ denote the ideal of $\widetilde{\mathbf{T}}_{n}$ defined in Lemma 3.1, By Lemma 2.8, this ideal is proper.

By Lemma 3.16 of [CG18], there is a surjective map

$$
\widetilde{R}_{n}^{\dagger} \rightarrow \widetilde{\mathbf{T}}_{n, \mathfrak{m}} \rightarrow \widetilde{S}:=\mathbf{T}_{\mathfrak{m}}\left[U_{p}\right] /\left(U_{p}^{2}-T_{p} U_{p}+\langle p\rangle\right)
$$

(which sends $T_{l}$ and $\langle l\rangle$ to $T_{l}$ and $\langle l\rangle$ respectively, and sends $U_{p}$ to $U_{p}$, where $U_{p}$ in $\widetilde{S}$ is viewed as a formal variable satisfying the given quadratic relation). Although the running assumption in $\S 3$ of [CG18] is that $p>2$, the proof of [CG18, Lemma 3.16] applies (as written with no changes necessary) with $p=2$. The image $S$ of $R_{n}^{\dagger} \subset \widetilde{R}_{n}^{\dagger}$ is generated by the values of $T$ and $D$ on Frobenius elements, which land inside the ring $\mathbf{T}_{\mathfrak{m}}$ (in fact, they generate the ring $\mathbf{T}_{\mathfrak{m}}$ ). But $\widetilde{S}$ is free of rank two over $\mathbf{T}_{\mathfrak{m}}$, and thus $\widetilde{S} / S$ has no annihilator. Consequently, the corresponding determinant in $\mathbf{T}_{\mathfrak{m}}$ is unramified by Proposition 2.10. To show that $T\left(\right.$ Frob $\left._{p}\right)=T_{p}$ and $D\left(\right.$ Frob $\left._{p}\right)=\langle p\rangle$, it suffices to show that $T(\phi)=T_{p}$ and $D(\phi)=$ $\langle p\rangle$. The image of $\alpha$ in $\mathbf{T}_{\mathfrak{m}}\left[U_{p}\right] /\left(U_{p}^{2}-T_{p} U_{p}+\langle p\rangle\right)$ was $U_{p}$, which satisfies the equation $X^{2}-T_{p} X+\langle p\rangle=0$. Yet $\alpha$ also satisfies the equation $X^{2}-T(\phi) X+D(\phi)=0$. Since this algebra is free of rank two over $\mathbf{T}_{\mathfrak{m}}$, these quadratics must be the same, and hence $T(\phi)=T_{p}$ and $D(\phi)=\langle p\rangle$.

Remark 3.3. The proof above relies on [CG18, Lemma 3.16]. We also note, however, that the content of this lemma is simply an alternate form of doubling which is a already implicit in the work of Wiese [Wie14].

Remark 3.4. One should also be able to apply the methods of this paper in the case $l \neq p$ when $l$ exactly divides $N$, where now one wants to capture in this context the notion of a determinant "admitting an unramified quotient line" when restricted to the inertia group $I_{l}$ at $l$ (cf. $\S 1.8$ of [WW18] $)$.

\section{REFERENCES}

[AL78] A. O. L. Atkin and Wen Ch'ing Winnie Li, Twists of newforms and pseudo-eigenvalues of $W$ operators, Invent. Math. 48 (1978), no. 3, 221-243. MR 508986

[Buz14] Kevin Buzzard, Computing weight one modular forms over $\mathbb{C}$ and $\overline{\mathbb{F}}_{p}$, Computations with modular forms, Contrib. Math. Comput. Sci., vol. 6, Springer, Cham, 2014, pp. 129-146. MR 3381451

[Cal18] Frank Calegari, Non-minimal modularity lifting in weight one, J. Reine Angew. Math. 740 (2018), 41-62. MR 3824782

[Car94] Henri Carayol, Formes modulaires et représentations galoisiennes à valeurs dans un anneau local complet, Contemporary Mathematics 165 (1994), 213-213.

[CE98] Robert F. Coleman and Bas Edixhoven, On the semi-simplicity of the $U_{p}$-operator on modular forms, Math. Ann. 310 (1998), no. 1, 119-127. MR 1600034

[CG18] Frank Calegari and David Geraghty, Modularity lifting beyond the Taylor-Wiles method, Invent. Math. 211 (2018), no. 1, 297-433. MR 3742760

[Che14] Gaëtan Chenevier, The p-adic analytic space of pseudocharacters of a profinite group and pseudorepresentations over arbitrary rings, Automorphic forms and Galois representations. Vol. 1, London Math. Soc. Lecture Note Ser., vol. 414, Cambridge Univ. Press, Cambridge, 2014, pp. 221-285. MR 3444227

[DDT97] Henri Darmon, Fred Diamond, and Richard Taylor, Fermat's last theorem, Elliptic curves, modular forms \& Fermat's last theorem (Hong Kong, 1993), Int. Press, Cambridge, MA, 1997, pp. 2-140. MR 1605752 (99d:11067b) 
[Del71] Pierre Deligne, Formes modulaires et représentations l-adiques, Séminaire Bourbaki. Vol. 1968/69: Exposés 347-363, Lecture Notes in Math., vol. 175, Springer, Berlin, 1971, pp. Exp. No. 355, 139-172. MR 3077124

[DS74] Pierre Deligne and Jean-Pierre Serre, Formes modulaires de poids 1, Ann. Sci. École Norm. Sup. (4) 7 (1974), 507-530 (1975). MR 0379379

[Edi06] Bas Edixhoven, Comparison of integral structures on spaces of modular forms of weight two, and computation of spaces of forms mod 2 of weight one, J. Inst. Math. Jussieu 5 (2006), no. 1, 134, With appendix A (in French) by Jean-François Mestre and appendix B by Gabor Wiese. MR 2195943 (2007f:11046)

[Gro90] Benedict H. Gross, A tameness criterion for galois representations associated to modular forms $(\bmod p)$, Duke Mathematical Journal 61 (1990), no. 2, 445-517.

[Kat73] Nicholas M. Katz, p-adic properties of modular schemes and modular forms, Modular functions of one variable, III (Proc. Internat. Summer School, Univ. Antwerp, Antwerp, 1972), Springer, Berlin, 1973, pp. 69-190. Lecture Notes in Mathematics, Vol. 350. MR 0447119

[Rou96] Raphaël Rouquier, Caractérisation des caractères et pseudo-caractères, J. Algebra 180 (1996), no. 2, 571-586. MR 1378546

[Sch15] George J. Schaeffer, Hecke stability and weight 1 modular forms, Math. Z. 281 (2015), no. 1-2, 159-191. MR 3384865

[Sno18] Andrew Snowden, Singularities of ordinary deformation rings, Math. Z. 288 (2018), no. 3-4, 759781. MR 3778977

[SW97] C. M. Skinner and A. J. Wiles, Ordinary representations and modular forms, Proc. Nat. Acad. Sci. U.S.A. 94 (1997), no. 20, 10520-10527. MR 1471466

[Tay91] Richard Taylor, Galois representations associated to Siegel modular forms of low weight, Duke Math. J. 63 (1991), no. 2, 281-332. MR 1115109

[WE18] Carl Wang-Erickson, Algebraic families of Galois representations and potentially semi-stable pseudodeformation rings, Math. Ann. 371 (2018), no. 3-4, 1615-1681. MR 3831282

[Wie14] Gabor Wiese, On Galois representations of weight one, Doc. Math. 19 (2014), 689-707. MR 3247800

[Wil88] A. Wiles, On ordinary $\lambda$-adic representations associated to modular forms, Invent. Math. 94 (1988), no. 3, 529-573. MR 969243

[WW18] Preston Wake and Carl Wang-Erickson, The Eisenstein ideal with squarefree level, arXiv e-prints (2018), arXiv:1804.06400.

[WWE17] Preston Wake and Carl Wang-Erickson, Ordinary pseudorepresentations and modular forms, Proc. Amer. Math. Soc. Ser. B 4 (2017), 53-71. MR 3738092 